\title{
YAP Promotes Breast Cell Proliferation and Survival Partially through Stabilizing the KLF5 Transcription Factor
}

\author{
Xu Zhi, ${ }^{\dagger}$ Dong Zhao, ${ }^{\dagger}{ }^{\dagger}$ Zhongmei Zhou, ${ }^{*}$ \\ Rong Liu, ${ }^{*}$ and Ceshi Chen* \\ From the Key Laboratory of Animal Models and Human \\ Disease Mechanisms of the Chinese Academy of Sciences $\mathcal{E}$ \\ Yunnan Province, Kunming Institute of Zoology, Chinese \\ Academy of Sciences, Kunming, China; and the Center for \\ Cell Biology and Cancer Research, ${ }^{\dagger}$ Albany Medical College, \\ Albany, New York
}

The Yes-associated protein (YAP), an oncoprotein in the Hippo tumor suppressor pathway, regulates tumorigenesis and has been found in a variety of tumors, including breast, ovarian, and hepatocellular cancers. Although YAP functions through its WW domains, the YAP WW domain-binding partners have not yet been completely determined. With this study, we demonstrate that YAP functions partially through its binding to KLF5, a transcription factor that promotes breast cell proliferation and survival. YAP interacted with the KLF5 PY motif through its WW domains, preventing the $\mathrm{E} 3$ ubiquitin ligase WWP1 from ubiquitinating KLF5. Overexpression of the wild-type YAP but not the WW domain-mutated YAP up-regulated KLF5 protein levels and mRNA expression levels of KLF5 downstream target genes, including FGFBP1 (alias FGF-BP) and ITGB2. In addition, knockdown of YAP decreased expression levels of KLF5, FGF-BP, and ITGB2. Depletion of either YAP or KLF5 decreased breast cell proliferation and survival in MCF10A and SW527 breast cell lines, and stable knockdown of either YAP or KLF5 suppressed SW527 xenograft growth in mice. The YAP upstream kinase LATS1 suppressed the KLF5-FGF-BP axis, as well as cell growth through YAP signaling. Both YAP and KLF5 are coexpressed in estrogen receptor $\mathrm{ER} \alpha$-negative breast cell lines. These findings suggest that KLF5 could be an important transcription factor partner for YAP and may contribute to the Hippo pathway. (Am J Pathol 2012, 180:2452-2461; bttp:// dx.doi.org/10.1016/j.ajpath.2012.02.025)
The Yes-associated protein (YAP) is a transcriptional coactivator and the major downstream effector of the Hippo tumor suppressor pathway in mammalian cells., ${ }^{1,2} \mathrm{Nu}$ clear YAP promotes cell proliferation and survival through regulating gene expression. ${ }^{3,4}$ YAP is expressed in a wide array of tumors, including breast tumors, ${ }^{5}$ ovarian cancer, ${ }^{6}$ hepatocellular carcinoma, ${ }^{7,8}$ esophageal squamous cell carcinoma, ${ }^{9}$ and Ewing's sarcoma. ${ }^{10}$ Once YAP is phosphorylated by the LATS-MOB kinase complex, YAP stays in the cytoplasm through interaction with 14-3-3 proteins. ${ }^{11}$ Several YAP upstream negative regulators (including LATS, MOB, and MST) are tumor suppressors that are mutated or down-regulated in cancers. $^{1,2}$ Furthermore, the chromosome region $11 \mathrm{q} 22$ containing the YAP gene (YAP1) is frequently amplified in breast cancer. ${ }^{5}$ Overexpression of YAP has been shown to promote breast cell proliferation, survival, and migration, ${ }^{5,12,13}$ and YAP has also been shown to stimulate growth of the mouse liver ${ }^{4,14}$ and liver cancer. ${ }^{3}$ However, the mechanism by which YAP promotes cancer development remains unclear.

The YAP protein contains several domains: a TEAD binding domain (TBD), a 14-3-3 binding motif, WW domains (YAP2 contains two WW domains, and YAP1 contains one), and a transcriptional activation domain. ${ }^{1}$ The YAP2 protein is the evolutionarily conserved YAP; in the present study, all YAP was YAP2. Although YAP functions partially through binding to the TEAD transcription factors, ${ }^{15,16}$ YAP uses the WW domains to bind to PY motifcontaining transcription factors, including $\mathrm{p} 73,{ }^{17,18} \mathrm{EGR}$ -

Supported by a grant from Yunnan Province High-Profile Talents Program (2010Cl114) and the National Nature Science Foundation of China General Program (81072162, 81120108019, and U1132605).

Accepted for publication February 7, 2012.

X.Z. and D.Z. contributed equally to this work.

Supplemental material for this article can be found at http://ajp. amjpathol.org or at $h$ ttp://dx.doi.org/10.1016/j.ajpath.2012.02.025.

Address reprint requests to Ceshi Chen, Ph.D., Key Laboratory of Animal Models and Human Disease Mechanisms of Chinese Academy of Sciences \& Yunnan Province, Kunming Institute of Zoology, Chinese Academy of Sciences, Kunming, Yunnan, 650223, China. E-mail: chenc@mail.kiz.ac.cn. 
Table 1. siRNA and shRNA Oligonucleotides Used in This Study

\begin{tabular}{lc}
\hline Target gene & Sequence \\
\hline $\begin{array}{l}\text { Luciferase siRNA/ } \\
\text { shRNA }\end{array}$ & $5^{\prime}$-CUUACGCUGAGUACUUCGA-3' \\
KLF5 siRNA & 5'-AAGCUCACCUGAGGACUCA-3' \\
KLF5 shRNA & 5'-CGAUUACCCUGGUUGCACA-3' \\
YAP SiRNA/shRNA & 5'-GGUGAUACUAUCAACCAAA-3' \\
LATS1 siRNA\#1 & 5'-GCCUUGCAGGAAAUUCGAA-3' \\
LATS1 SiRNA\#2 & 5'-CCUCCAUACGAGUCAAUCA-3' \\
\hline
\end{tabular}

$1,{ }^{19}$ ErbB4 $4{ }^{20-22}$ and RUNX2. ${ }^{23}$ The WW domains are essential for YAP to regulate a subset of gene expressions and to stimulate growth and oncogenic transformation. ${ }^{13,24}$ However, the key proproliferative and prosurvival YAP partner transcription factor has not been determined. ${ }^{13}$

The KLF5 transcription factor is highly expressed in estrogen receptor-negative $\left(E R \alpha^{-}\right)$basal-type poorly differentiated breast cancer. ${ }^{25} \mathrm{~A}$ high level of KLF5 mRNA expression is an unfavorable prognostic marker, correlated with shorter survival for breast cancer patients. ${ }^{26}$ We have previously demonstrated that overexpression of KLF5 promotes cell proliferation, survival, and tumor growth predominately through a downstream oncogenic target gene, FGFBP1 (alias FGF-BP). ${ }^{27-29}$ Like many oncogenic transcription factors, KLF5 activity is tightly regulated at the protein post-translational level. ${ }^{30}$ We have previously shown that KLF5 is ubiquitinated by two E3 ubiquitin ligases (WWP1 and SCF ${ }^{\mathrm{Fbw}}$ ). ${ }^{31,32}$ WWP1 binds to the KLF5 PY motif through its WW domains and targets KLF5 for degradation. However, how KLF5 protein stability is positively regulated has not previously been reported.

In theory, YAP could bind to KLF5 through the WW domain/PY motif interaction. Both YAP and KLF5 are oncoproteins promoting cell division, survival, and embryonic stem cell self-renewal. ${ }^{33,34}$ We therefore hypothesized that KLF5 is a YAP partner transcription factor for breast cell proliferation and survival. Here we demonstrate that KLF5 is regulated by the Hippo tumor suppressor pathway. Our findings provide valuable insights into the regulatory mechanism of KLF5 in breast cancer.

\section{Materials and Methods}

\section{Cell Lines, Cell Culture and Transient Transfection}

Thirteen breast cell lines were investigated by Western blotting: 2 primary cell lines (184 and 48RS), 3 immortalized cell lines (MCF10A, 184A1, and 184B5), $4 \mathrm{ER} \alpha^{+}$ cancer cell lines (MCF7, HCC1500, MDA-MB-134, and T47D), and $4 E R \alpha^{-}$cancer cell lines (SW527, HCC1937, HCC1806, and SUM149PT). All cell lines were purchased from ATCC (Manassas, VA).

The SW527 breast cancer cell line was maintained in Dulbecco's modified Eagle's medium, supplemented with $5 \%$ fetal bovine serum, $4.5 \mathrm{~g} / \mathrm{L}$ glucose, $1.5 \mathrm{~g} / \mathrm{L}$ sodium bicarbonate, and $1 \%$ penicillin/streptomycin. The
MCF10A immortalized breast cell line, the TSU-Pr1 bladder cancer cell line-derived clones $\mathrm{K} 12$ and $\mathrm{V} 4$, the 22Rv1 prostate cancer cell line, and the human embryonic kidney cell line HEK293T were cultured as described previously. 27,35,36 Lipofectamine 2000 (Invitrogen, Carlsbad, CA) was used for the transient transfection for siRNAs (20 nmol/L final concentration) and plasmids (1.6 $\mu \mathrm{g}$ in 12-well plate). The siRNA sequences for YAP, KLF5, LATS1, and luciferase genes are listed in Table 1. Cells were harvested at 48 hours after transfection.

\section{Retrovirus and Lentivirus Construction}

Wild-type YAP $\left(\mathrm{YAP}_{\mathrm{wt}}\right)$ and two WW-domain mutated YAP $\left(Y_{A} P_{w 1 w 2}\right)^{13}$ were subcloned into the pBabe-puro retroviral vector. All plasmids were transfected into AMP293 packaging cells to produce retroviruses. The $\mathrm{pSIH} 1-\mathrm{H} 1$ puro shRNA vector (System Biosciences, Mountain View, CA) was used to express YAP shRNA, KLF5 shRNA, and luciferase (LUC) shRNA. Target sequences for LUC, YAP, and KLF5 are listed in Table 1.

\section{Quantitative RT-qPCR}

Total RNAs were extracted using TRIzol reagent (Invitrogen). Reverse transcription was performed with random hexanucleotide primers using an iScript cDNA synthesis kit (Bio-Rad, Hercules, CA). Quantitative PCR (qPCR) was performed using a FastStart SYBR Green master mix with Rox (Roche Diagnostics, Indianapolis, IN). Primers used are listed in Table 2.

\section{Antibodies}

The anti-YAP ( $\mathrm{H}-125)$ rabbit polyclonal (sc-15407), anti-HA (Y-11) rabbit polyclonal (sc-805), and anti-Myc (9E10) mouse monoclonal (sc-40) antibodies were from Santa Cruz Biotechnology (Santa Cruz, CA). The rabbit polyclonal anti-KLF5 and anti-WWP1 antibodies have been described elsewhere. ${ }^{32}$ The anti-phospho-YAP (S127) rabbit polyclonal (4911), anti-PARP rabbit polyclonal (9542), anti-caspase-3 rabbit polyclonal (9662),

Table 2. PCR Primers Used in This Study

\begin{tabular}{|c|c|}
\hline Name & Primer sequence \\
\hline & RT-qPCR \\
\hline KLF5-Fwd & $5^{\prime}-\mathrm{ACACCAGACCGCAGCTCCA}-3^{\prime}$ \\
\hline KLF5-Rev & $5^{\prime}$-TCCATTGCTGCTGTCTGATTTGTAG-3' \\
\hline FGF-BP-Fwd & $5^{\prime}-$ TGTTCAGAGGCTGTTTCCTG-3' \\
\hline FGF-BP-Rev & 5'-TTCAGCAGAAAGTTCGTTGC-3' \\
\hline ITGB2-Fwd & 5'-AAGTGACGCTTTACCTGCGA-3' \\
\hline ITGB2-Rev & 5'-CCTGAGGTCATCAAGCATGG-3' \\
\hline GAPDH-Fwd & 5'-GGTGAAGGTCGGAGTCAACG-3' \\
\hline GAPDH-Rev & 5' -TGGGTGGAATCATATTGGAACA-3' \\
\hline \multicolumn{2}{|c|}{ Cloning YAP into the pBabe-puro vector } \\
\hline YAP-Fwd & $\begin{array}{l}5^{\prime} \text {-TTGAATTCACCATGGATCCCGGGCAG } \\
\text { CAGCC- } 3^{\prime}\end{array}$ \\
\hline YAP-Rev & $\begin{array}{l}5^{\prime}-\text { TTTGTCGACCTATAACCATGTAAGA } \\
\text { AAGC-3' }\end{array}$ \\
\hline
\end{tabular}


and anti-LATS1 (C66B5) rabbit monoclonal (3477) antibodies were from Cell Signaling Technology (Danvers, MA). The anti-FLAG rabbit polyclonal (F7425), mouse monoclonal anti-YAP1 antibody (SAB1404823), and anti$\beta$-actin (AC-15) mouse monoclonal (A5441) antibodies were from Sigma-Aldrich (St. Louis, MO). The goat antiKLF5 and mouse anti-FGF-BP monoclonal antibodies (MAB1593) were from R\&D Systems (Minneapolis, MN). The rabbit anti-Ki-67 antibody (RM-9106-S0) was from Thermo Lab Vision (Kalamazoo, MI).

\section{Immunoprecipitation, Immunofluorescence, and KLF5 Ubiquitination}

Immunoprecipitation, immunofluorescence, and KLF5 ubiquitination assays were performed as described previously. ${ }^{30,31}$

\section{Cell Proliferation and Viability Assays}

Cell proliferation of MCF10A and SW527 cells was measured by the ${ }^{3} \mathrm{H}$-thymidine incorporation assay, as described previously. ${ }^{27,35}$ Cell viability was measured using the sulforhodamine $B(\mathrm{SRB})$ assay. ${ }^{35}$

\section{Tumorigenesis in NOD SCID Mice}

Eighteen NOD SCID female mice, 4 to 5 weeks old, were purchased from Charles River Laboratories International (Wilmington, DE). The mice were randomly distributed equally into three groups: $L U C_{s h}, K L F 5_{s h}$, and $\mathrm{YAP}_{\text {sh. }}$. SW527 cells were subcutaneously injected into the flank of both sides $\left(2.5 \times 10^{6}\right.$ cells per site). Tumor volumes were measured twice a week from day 9 and were calculated as $1 / 2$ (length $\times$ width $^{2}$ ). All mice were sacrificed at day 24 , and tumor wet weights were measured. Xenografts were fixed and stained for $\mathrm{Ki}-67$ and cleaved caspase-3 using standard $\mathrm{IHC}$ protocols.

\section{Statistical Analysis}

DNA synthesis, cell viability, anchorage-independent growth, and $\mathrm{IHC}$ assays were conducted in triplicate. Data were reported as means $\pm S D$, and differences between any two groups were compared using $t$-tests. $P$ values of $<0.05$ were considered significant.

\section{Results}

\section{YAP Interacts with KLF5 through the WW Domains and PY Motif}

To test whether endogenous YAP interacts with KLF5, a coimmunoprecipitation experiment was performed in MCF10A cells (Figure 1A). When the endogenous KLF5 protein was immunoprecipitated by the goat anti-KLF5 antibody, the endogenous YAP protein could be detected in the complex. The goat IgG control immunoprecipitated neither KLF5 nor YAP. These results suggest that endogenous YAP and KLF5 proteins specifically interact with each other.
A
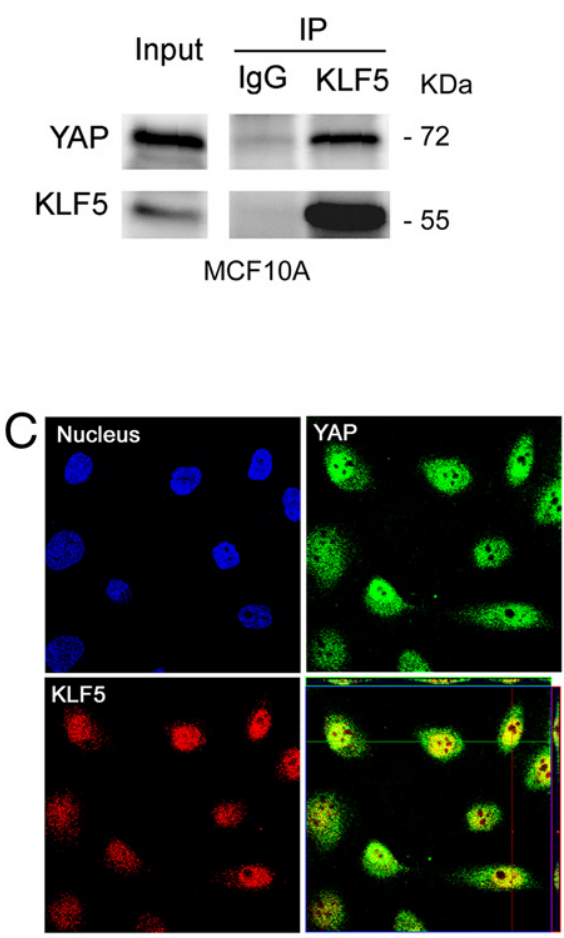

MCF10A

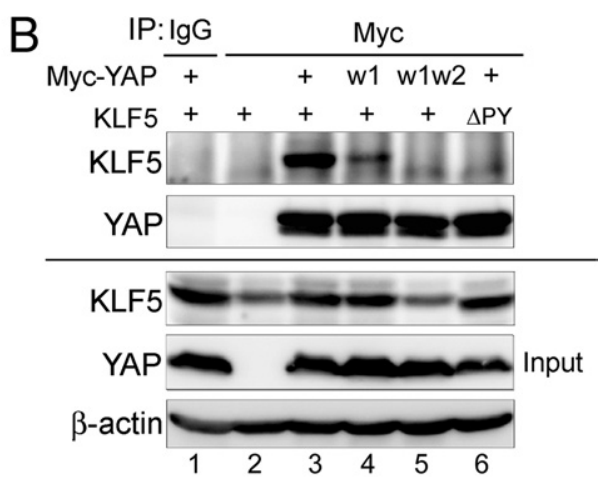

D

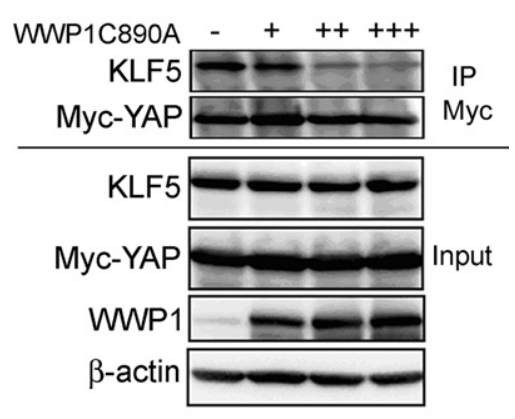

Figure 1. The YAP protein interacts with KLF5 through the WW domains and PY motif. A: Coimmunoprecipitation between endogenous YAP and KLF5 proteins in MCF10A cells. Goat antihuman KLF5 antibody was used for immunoprecipitation; goat IgG was used as the negative control. B: Protein interaction between YAP and KLF5 proteins depends on the WW domains and PY motif. HEK293T cells were transfected with different YAP and KLF5 constructs. Anti-Myc antibody was used for immunoprecipitation mouse IgG was used as the negative control $\mathrm{YAP}_{\mathrm{wt}}$ and $\mathrm{YAP}_{\mathrm{w} 1}$ (in which the first WW domain is mutated) increased KLF5 protein levels. $\mathrm{YAP}_{\mathrm{w} 1 \mathrm{w} 2}$ (with WW domains mutated) did not increase the KLF5 protein level. Lane $1, \mathrm{YAP}_{\mathrm{wt}}$; lane 2, empty vector; lane $3, \mathrm{YAP}_{\mathrm{wt}}$; lane 4 $\mathrm{YAP}_{\mathrm{w} 1}$; lane 5, YAP ${ }_{\mathrm{w} 1 \mathrm{w} 2}$; lane 6, YAP ${ }_{\mathrm{wt}}$. C: The nuclear colocalization of endogenous KLF5 and YAP in MCF10A cells was determined by immunofluorescence. Cells were fixed and stained with the anti-YAP and anti-KLF5 antibodies. Alexa Fluor 488 goat anti-mouse secondary antibody was used to stain YAP, and rhodamine goat anti-rabbit secondary antibody was used to stain KLF5. Nuclei were stained with DAPI. KLF5 proteins are observed in the nucleus. D: YAP and WWP1 compete for interaction with KLF5. The Myc-YAP and KLF5 protein complexes decreased with an increase of WWP1C890A. Different amounts $(0, \quad 0.375, \quad 0.75, \quad 1.5 \mu \mathrm{g})$ of WWP1C890A plasmids were cotransfected with KLF5 and Myc-YAP into HEK293T cells. Anti-Myc antibody was used for immunoprecipitation. 
To determine whether both WW domains of YAP are required for the interaction, KLF5 and several Myctagged YAP (WT, w1 mutant, and w1w2 mutant) ${ }^{13}$ proteins were coexpressed in HEK293T cells. The WT and w1 mutated YAP proteins increased KLF5 protein levels, compared with an empty vector; the w1w2 mutated YAP had no effect on KLF5 protein levels (Figure 1B). When all Myc-YAP proteins were immunoprecipitated with a similar efficiency, KLF5 was coimmunoprecipitated with WT YAP and to a lesser extent with $Y A P_{w 1}$, but not with $Y A P_{w 1}{ }_{w 2}$. The control IgG immunoprecipitated neither YAP nor KLF5. Additionally, $\mathrm{YAP}_{\mathrm{w} 2}$ did not interact with KLF5 and stabilize KLF5 (see Supplemental Figure S1 at http://ajp.amjpathol.org). These findings suggest that YAP may stabilize KLF5 after binding to KLF5 predominately through the second WW domain, although the first WW domain appears to play a minor role. Additionally, KLF5 $\mathrm{PPY}$ is a more stable protein than WT KLF5 (Figure 1B), because this mutant is resistant to WWP1-mediated degradation. ${ }^{31}$ When cotransfected with WT Myc-YAP, KLF5 $\Delta$ PY could not be coimmunoprecipitated with Myc-YAP (Figure 1B), suggesting that the KLF5 PY motif is required for the interaction.

To determine whether KLF5 and YAP are colocalized in cells, we examined the subcellular localizations of YAP and KLF5 in subconfluent MCF10A cells by immunofluorescence. YAP was localized in both the nucleus and cytoplasm, as observed under confocal microscopy. KLF5 was localized in the nucleus. Nuclear colocalization of YAP and KLF5 was clearly evident (Figure 1C).

Given that KLF5 interacts with both YAP and WWP1 through the unique PY motif, the KLF5-YAP and KLF5WWP1 protein complexes should be exclusive of each other; that is, YAP and WWP1 may compete for interaction with KLF5. To test this possibility, different amounts of WWP1C890A, a catalytic inactive mutant of WWP1 that cannot degrade KLF5, were cotransfected with KLF5 and YAP. As expected, the KLF5-YAP protein complex was gradually decreased with the increase of WWP1C890A (Figure 1D). WWP1 itself did not interact with YAP (see Supplemental Figure S2 at $h$ ttp://ajp.amjpathol.org). These results suggest that YAP and WWP1 can bind exclusively to KLF5 and that YAP may protect KLF5 from WWP1-induced degradation.

\section{YAP Protects the KLF5 Protein from WWP1- Mediated Ubiquitination and Degradation}

It is unknown whether endogenous YAP stabilizes KLF5 in breast cells, although overexpression of YAP increased KLF5 protein levels in HEK293T cells (Figure 1B). To test this possibility, we knocked down YAP by siRNA in MCF10A cells. The KLF5 protein level was decreased

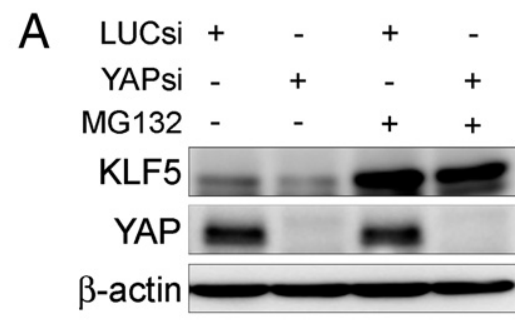

MCF10A
B

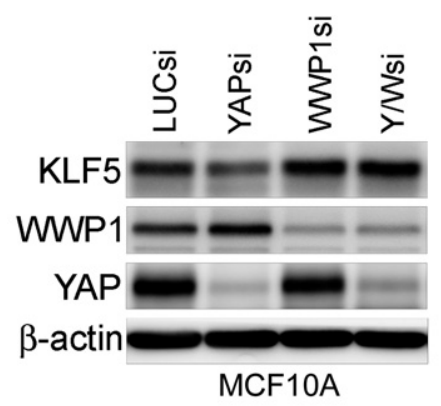

C

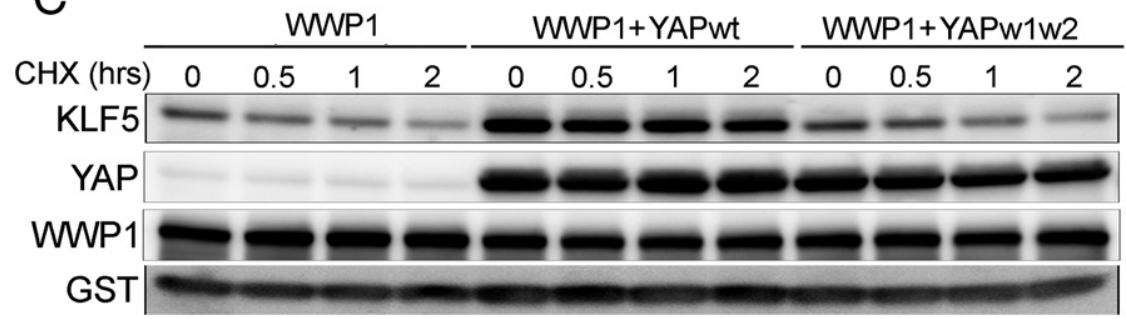

Figure 2. YAP protects the KLF5 protein from WWP1-mediated ubiquitination and degradation. A: Endogenous YAP decreased KLF5 protein degradation by the proteasome. MCF10A cells were transfected with the luciferase control siRNA and YAP siRNA. As necessary, the proteasome inhibitor MG132 $(20 \mu \mathrm{mol} / \mathrm{L})$ was added for 4 hours before harvest. The relative KLF5 protein levels were quantified (see Supplemental Figure S3A at http://ajp.amjpathol.org). B: Endogenous YAP decreased KLF5 protein degradation by WWP1. KLF5 protein levels were not down-regulated by the YAP siRNA when WWP1 was depleted. For quantitative data, see Supplemental Figure S4A (available at http://ajp. amjpathol.org). C: $\mathrm{YAP}_{\mathrm{wt}}$ but not $\mathrm{YAP}_{\mathrm{w} 1 \mathrm{w} 2} \mathrm{ex}-$ tended the KLF5 half-life in the presence of WWP1 in HEK293T cells. The half-lives of KLF5 were measured by the cycloheximide chase assay. For quantitative data, see Supplemental Figure S4B (available at http://ajp.amjpathol.org). D: YAP blocked WWP1-mediated KLF5 ubiquitination. 22Rv1 cells were transfected with KLF5$3 \times$ FLAG, WWP1, HA-Ub, YAP ${ }_{\mathrm{wt}}$, and $\mathrm{YAP}_{\mathrm{w} 1 \mathrm{w} 2}$. The ubiquitinated KLF5-3×FLAG proteins were immunoprecipitated with anti-FLAG M2 beads under a denaturing condition and were detected by the anti-HA antibody. 
A

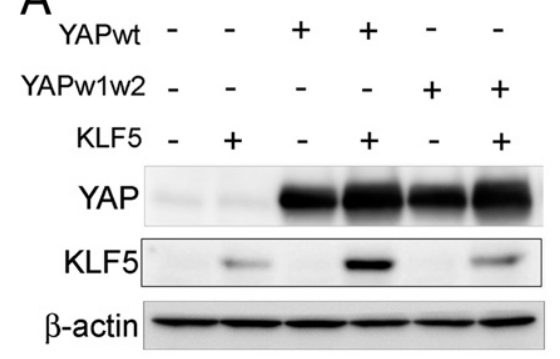

B
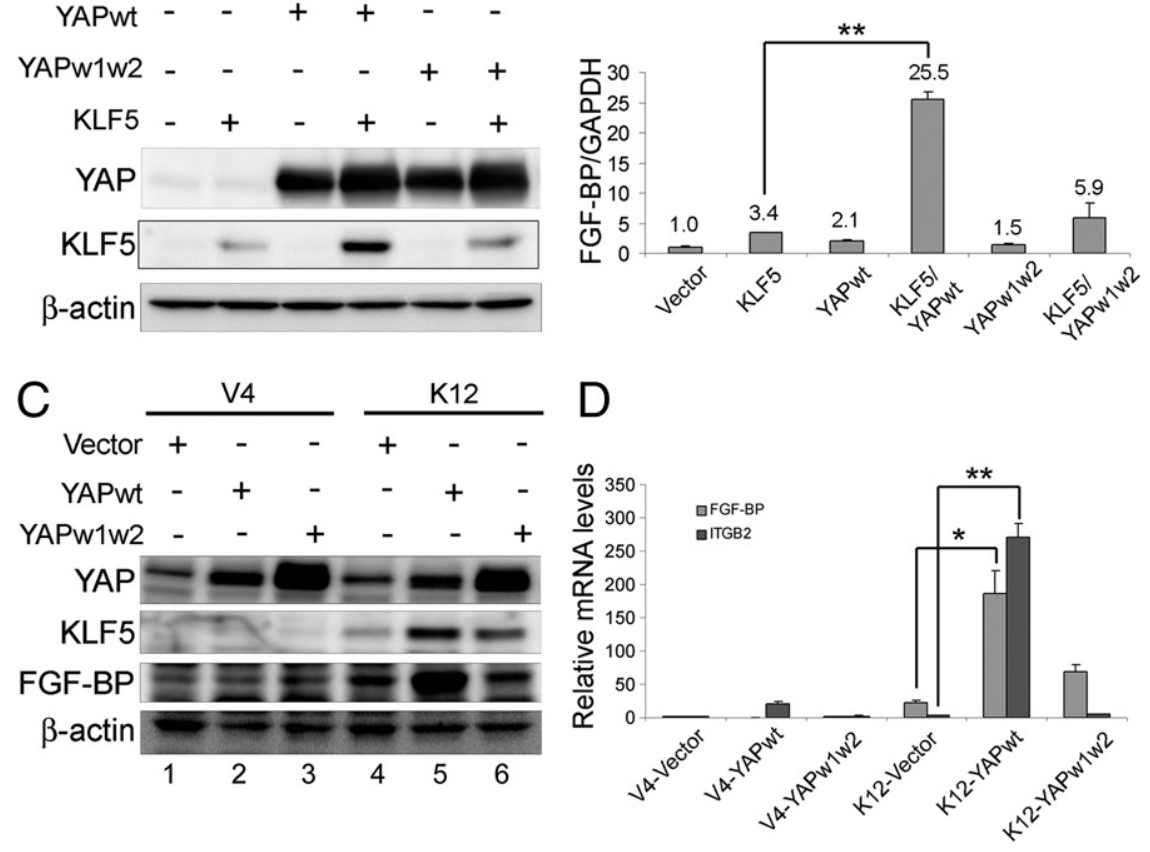

Figure 3. YAP overexpression increases the KLF5 protein level and promotes its target gene expression. A: Overexpression of $\mathrm{YAP}_{\mathrm{wt}}$, but not $\mathrm{YAP}_{\mathrm{w} 1 \mathrm{w} 2}$, increased KLF5 expression level HEK293T cells were transiently transfected with KLF5 and $\mathrm{YAP}_{\mathrm{wt}}$ or $\mathrm{YAP}_{\mathrm{w} 1 \mathrm{w} 2}$. Expression levels of KLF5 and YAP were detected by Western blotting. B: Overexpression of $\mathrm{YAP}_{\mathrm{wt}}$, but not $\mathrm{YAP}_{\mathrm{w} 1 \mathrm{w} 2}$, significantly $(P=0.002)$ increased KLF5-mediated FGFBP1 mRNA expression in HEK293T cells. The relative FGFBP1 mRNA levels were examined by RT-qPCR, using GAPDH as the internal control. C: Stable overexpression of $\mathrm{YAP}_{\mathrm{wt}}$, but not $\mathrm{YAP}_{\mathrm{w} 1 \mathrm{w} 2}$, increased KLF5 and FGF-BP protein expression levels in TSU-Pr1-K12 cells. K12 is a stable isogenic TSU-Pr1 clone overexpressing KLF5; V4 is the negative control clone. $\mathrm{YAP}_{\mathrm{wt}}$ and $\mathrm{YAP}_{\mathrm{w} 1 \mathrm{w} 2}$ were stably expressed by the pBabe-puro retroviral system. D: Stable overexpression of YAP but not $\mathrm{YAP}_{\mathrm{w} 1 \mathrm{w} 2}$, increased KLF5-mediated FGFBP1 $(P=0.02)$ and ITGB2 $(P=0.003)$ mRNA levels, as determined by RT-qPCR. GAPDH was used as the internal control.

when YAP expression was depleted (Figure 2A; see also Supplemental Figure S3 at http://ajp.amjpathol.org). We note that the KLF5 mRNA level did not decrease (see Supplemental Figure S3B at http://ajp.amjpathol.org). Similar results were observed in the SW527 breast cancer cell line (see Supplemental Figure S3C at http://ajp. amjpathol.org). MG132, a proteasome inhibitor, has previously been shown to increase the KLF5 protein level in MCF10A cells, ${ }^{30}$ and partially rescue the decrease of KLF5 by the YAP siRNA in MCF10A cells (Figure 2A; see also Supplemental Figure S3A at $h$ ttp://ajp.amjpathol.org), suggesting that endogenous YAP stabilizes the KLF5 protein by preventing proteasomal degradation.

If YAP stabilizes KLF5 by preventing WWP1 from degrading KLF5, the YAP siRNA should have no effect in the absence of WWP1. Indeed, when endogenous WWP1 was depleted by siRNA, the KLF5 protein level was upregulated (1.4-fold) in MCF10A cells (Figure 2B; see also Supplemental Figure S4A at http://ajp.amjpathol.org). Further depletion of YAP did not down-regulate KLF5 further (Figure 2B); thus, YAP and WWP1 are antagonist, and the expression of YAP and WWP1 together determine KLF5 protein levels in MCF10A cells.

To further determine whether YAP protects KLF5 from WWP1-mediated degradation, KLF5 and WWP1 were coexpressed with YAP (empty vector, WT, and the w1w2 mutant) in HEK293T cells, and the KLF5 protein half-lives were measured by the cycloheximide chase assay (Figure $2 \mathrm{C}$ ). Consistent with previous results (Figure 1B), the KLF5 steady levels and half-lives were specifically elevated and extended by YAP $_{\text {wt }}$ but not by WW domain mutated YAP w1w2 $_{2}$ (Figure 2C; see also Supplemental Figure S4B at http://ajp.amjpathol.org). Consistently, $\mathrm{YAP}_{\mathrm{wt}}$ but not $\mathrm{YAP}_{\mathrm{w} 1 \mathrm{w} 2}$ decreased the KLF5 ubiquitination by WWP1 in 22Rv1 cells (Figure 2D). Taken together, these results demonstrate that YAP interacts with KLF5 in a WW domain-dependent manner. Furthermore, YAP decreases the binding between KLF5 and WWP1 that subsequently decreases KLF5 ubiquitination and degradation.

\section{YAP Overexpression Promotes KLF5 Target Gene Expression}

To investigate whether YAP increases KLF5 activities, YAP and KLF5 were transiently overexpressed in HEK293T cells. When the KLF5 protein level was specifically elevated by $Y_{A} P_{w t}$ but not by $Y_{A} P_{w 1 w 2}$ (Figure 3A), the mRNA level of FGFBP1, a well established KLF5 target gene, ${ }^{28}$ was significantly increased by $Y_{A P}$ wt but not by $\mathrm{YAP}_{w 1 w 2}$, as determined by RT-qPCR (Figure 3B).

Previously we reported that KLF5 induced FGFBP1 expression in TSU-Pr1 cells. ${ }^{27}$ To validate the results in HEK293T cells, YAP ${ }_{\text {wt }}$ and $Y A P_{\text {w1w2 }}$ were stably expressed in TSU-Pr1-V (clone V4) and TSU-Pr1-KLF5 (clone K12) cells (Figure $3 \mathrm{C}$ ). Consistently, $\mathrm{YAP}_{\mathrm{wt}}$ but not $\mathrm{YAP}_{\mathrm{w} 1 \mathrm{w2}}$ increased KLF5-mediated FGFBP1 induction. The induction of FGFBP1 occurred at the mRNA level (Figure 3D). A YAP WW domain-dependent target gene $/ T G B 2^{13}$ was also induced by KLF5 and YAP in a similar fashion as FGFBP1 (Figure 3D). These data suggest that YAP can increase the KLF5 activity of inducing downstream gene expression.

\section{YAP Knockdown Decreases KLF5 Target Gene Expression and Suppresses Breast Cell Proliferation, Survival, and Tumorigenesis}

To investigate whether endogenous YAP regulates endogenous KLF5 functions, we knocked down YAP and KLF5 individually by siRNA in MCF10A cells. Knockdown of YAP not only decreased the KLF5 protein level but also 


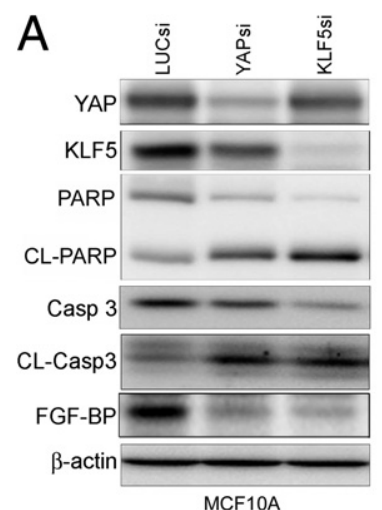

\section{B}
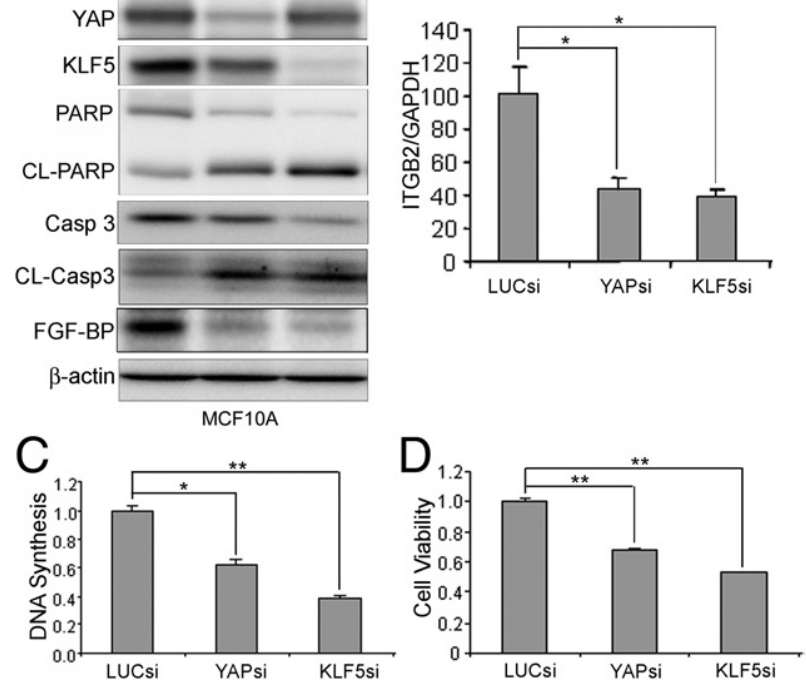

Figure 4. YAP knockdown decreases KLF5 target gene expression an suppresses cell proliferation and survival in MCF10A cells. A: Transient knockdown of YAP and KLF5 by siRNA decreased FGF-BP protein expression levels and induced apoptosis, as determined by PARP and caspase-3 cleavage. B: ITGB2 mRNA levels were significantly decreased when YAP and KLF5 were knocked down by siRNA in MCF10A, as examined by RT-qPCR Luciferase siRNA was used as the negative control. C: YAP and KLF5 knockdown significantly decreased DNA synthesis of MCF10A cells. DNA synthesis was examined by the ${ }^{3} \mathrm{H}$-thymidine incorporation assay. D: YAP and KLF5 knockdown significantly decreased the MCF10A cell viability, as determined by the SRB assay. ${ }^{*} P<0.05 ;{ }^{* *} P<0.01 t$-test.

decreased the FGF-BP protein level (Figure 4A). Knockdown of either YAP or KLF5 significantly decreased the ITGB2 mRNA expression level (Figure 4B). Similar results were observed in SW527 cells (see Supplemental Figure S4C at http://ajp.amjpathol.org).

Previously we have demonstrated that knockdown of KLF5 in the MCF10A cell line suppressed cell proliferation and induced apoptosis. ${ }^{29,32,37}$ Consistently, knockdown of YAP also significantly decreased DNA synthesis (Figure 4C) and induced apoptosis, as indicated by caspase-3 and PARP cleavage (Figure 4A). Consequently, knockdown of YAP significantly decreased MCF10A cell viability (Figure 4D).

To further determine the role of YAP and KLF5 in breast cancer and to provide proof-of-concept evidence for targeting the YAP-KLF5 pathway for breast cancer, we stably knocked down YAP and KLF5 in SW527 cells. As expected, stable knockdown of YAP down-regulated KLF5 and FGF-BP protein levels (Figure 5A). Knockdown of either YAP or KLF5 significantly decreased SW527 DNA synthesis and cell viability (Figure 5C). Furthermore, knockdown of either YAP or KLF5 significantly reduced anchorage-independent growth in soft agar and tumor growth in NOD SCID mice (Figure 5B). In agreement with in vitro results, knockdown of either YAP or KLF5 significantly decreased mRNA expression of FGFBP1 and ITGB2 as determined by RT-qPCR (see Supplemental Figure S4D at http://ajp.amjpathol.org). Knockdown of either YAP or KLF5 significantly decreased cell proliferation, as determined by Ki-67 staining, and induced apoptosis, as determined by cleaved caspase-3 staining
(Figure 5D). These data suggest that both endogenous YAP and KLF5 promote breast cancer cell proliferation, survival, and tumor growth.

\section{KLF5 Is Suppressed by LATS1 through YAP in Breast Cells}

YAP has been shown to be suppressed by LATS1 but not LATS2 kinase in MCF10A cells. ${ }^{12,38}$ Given that YAP stabilizes KLF5, LATS1 should down-regulate KLF5 through YAP in breast cells. Indeed, when LATS1 was knocked down by two different siRNAs in MCF10A cells, the KLF5 and FGF-BP protein levels were up-regulated (Figure 6A; see also Supplemental Figure S5A at $h$ ttp://ajp.amjpathol. org). To further determine whether LATS1 suppresses KLF5-FGF-BP signaling axis through YAP, we knocked down LATS1, YAP, and KLF5 singly and in combination (Figure 6B). As expected, LATS1 depletion decreased the pYAP-S127 level. More importantly, the up-regulation of KLF5 and FGF-BP by LATS1 depletion was completely abrogated when YAP was depleted. Furthermore, LATS1 depletion increased cell viability in the MCF10A cell line,

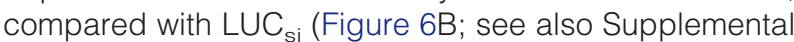
Figure S5B at http://ajp.amjpathol.org). When YAP or KLF5 was silenced, LATS1 $1_{\mathrm{si}}$-induced cell viability increase was completely blocked. These results indicate that LATS1 suppresses the KLF5-FGF-BP signaling axis and cell proliferation through YAP in MCF10A cells.

\section{Both YAP and KLF5 Are Predominately Expressed in $E R \alpha^{-}$Breast Cell Lines}

KLF5 has previously been shown to be expressed in a subset of ER $\alpha^{-}$breast cell lines. ${ }^{28,32}$ In contrast, WWP1 is expressed predominately in $\mathrm{ER} \alpha^{+}$breast cancers. ${ }^{32,39}$ The expression of YAP in different breast cell lines has not been compared previously, although it was detected in T47D, MDA-MB-231, and BT474 cell lines. ${ }^{40}$ In the present study, 13 breast cell lines were investigated by Western blotting. Of note, YAP was expressed predominately in $\mathrm{ER} \alpha^{-}$breast cell lines. In three of the four $\mathrm{ER} \alpha^{+}$ breast cancer cell lines (MCF7, HCC1500, and MDA-MB134), YAP protein expression was at very low levels (Figure $6 \mathrm{C}$ ). The expression pattern of YAP in these cell lines was very similar to that of KLF5 but opposite to that of WWP1.

\section{Discussion}

This study revealed a new positive regulatory mechanism for KLF5 at the protein post-translational level. KLF5 is a PY motif-containing transcription factor promoting breast cell proliferation and survival. Previously we had demonstrated that the E3 ubiquitin ligase WWP1 targets KLF5 for ubiquitin-mediated degradation through binding to the KLF5 PY motif. ${ }^{31}$ Here, we have shown that YAP also interacts with the KLF5 PY motif in a WW domain-dependent manner. In contrast to WWP1, YAP stabilized KLF5 and increased its activities of inducing target gene expression and promoting cell proliferation and survival. 
A

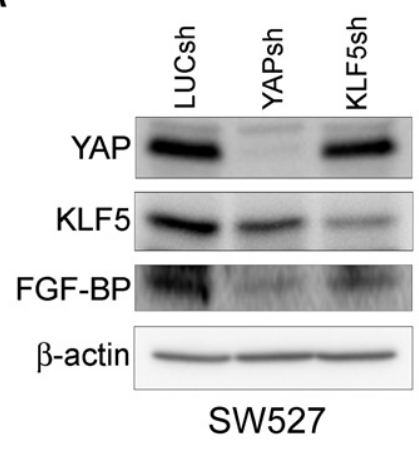

B

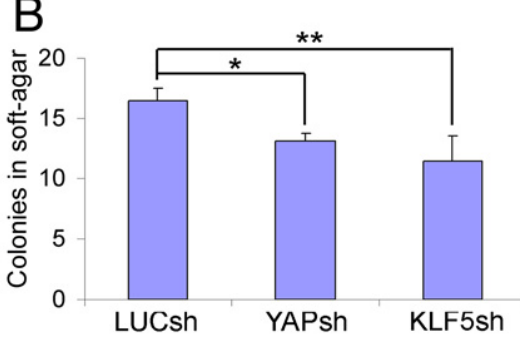

D

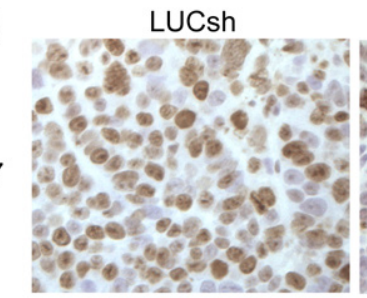

$59.9 \pm 4.4 \%$

YAPsh

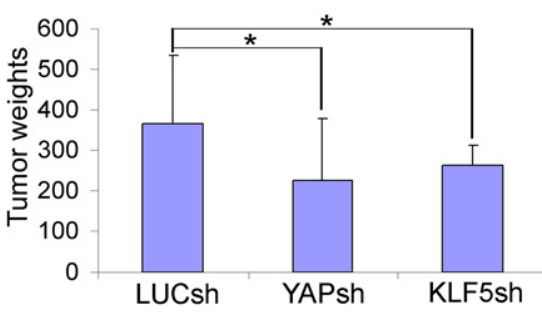

KLF5sh

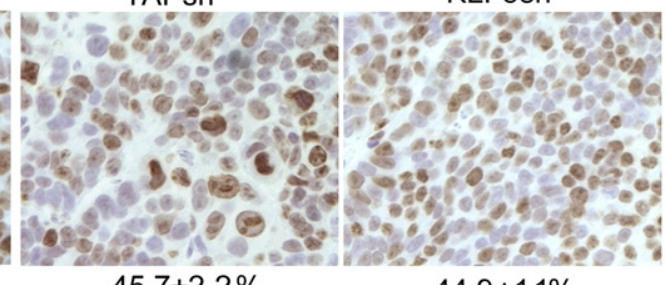

$45.7 \pm 2.2 \%$

$44.9 \pm 1.1 \%$

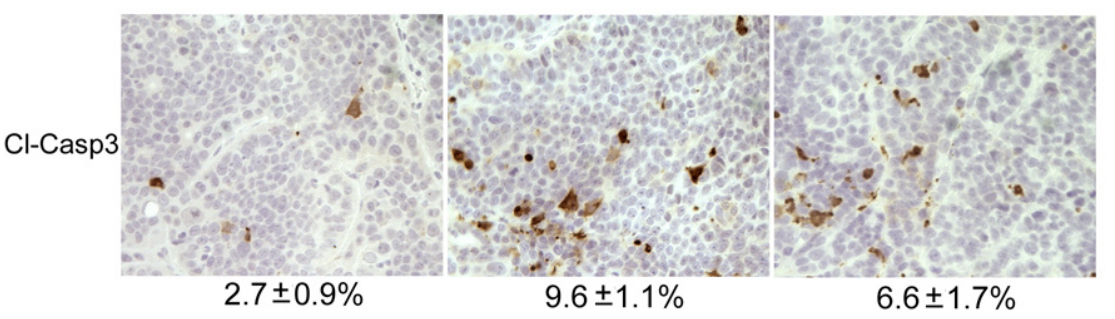

Figure 5. YAP knockdown in the SW527 breast cancer cell line decreases KLF5 target gene expression and suppresses cell proliferation, survival, and xenograft growth in mice. A: Stable knockdown of YAP and KLF5 by shRNA decreased FGF-BP protein levels. B: Stable knockdown of YAP and KLF5 by shRNA significantly decreased anchorage-independent growth in soft agar and xenograft tumor growth in NOD SCID mice. Average colony number per field and average tumor weight $(\mathrm{mg})$ are shown. C: Stable knockdown of YAP and KLF5 by shRNA significantly decreased DNA synthesis and cell viability. D: Stable knockdown of YAP and KLF5 by shRNA significantly decreases cell proliferation and survival, as measured by Ki-67 and cleaved caspase-3 IHC staining. Quantitative results are given below each panel (mean $\pm \mathrm{SD}$ ). There were significant differences between $\mathrm{LUC}_{\mathrm{sh}}$ and YAP $_{\text {sh }}(P=0.015 \mathrm{Ki}-67 ; P=0.002 \mathrm{CL}-\mathrm{C} 3)$ or $\mathrm{KLF}_{\text {sh }}(P=0.0095 \mathrm{Ki}-67 ; P=0.04 \mathrm{CL}-\mathrm{C} 3) .{ }^{*} P<$ $0.05 ;{ }^{* * * *} P<0.01 t$-test.
The major functional mechanism of YAP appears to be that YAP protects KLF5 from WWP1-mediated ubiquitination and degradation (Figure 6D). It is possible that KLF5 stability and activity are regulated also by other WW domain-containing proteins.

KLF5 is regulated by several signaling pathways, including the Ras-MAPK, ${ }^{41} \mathrm{Wnt}^{42}$ and TGF $\beta^{43}$ pathways. Here, we have demonstrated for the first time that KLF5 is regulated by the Hippo tumor suppressor pathway. LATS1 suppressed KLF5 functions through YAP (Figure 6). Thus, KLF5 could be a component of the Hippo tumor suppressor pathway that decreases cell proliferation and survival.

KLF5 may be an important YAP partner transcription factor in breast cells. As a transcriptional coactivator, YAP has also been reported to regulate the expression of a number of oncogenic target genes, including ITGB2, CTGF, ALPP, PIK3C2B, BIRC5 (alias survivin), and AREG in breast cells. ${ }^{13,44}$ It was believed that YAP regulates target gene transcription through different transcription factor partners, including TEAD, p73, ErbB4, and RUNX2. The essential role of WW domains for YAP oncogenic transformation activity suggests that the PY motif-containing transcription factor partners are significant. $^{13}$ As a PY motif-containing transcription factor, KLF5 has been shown to regulate the expression of many oncogenic target genes, including FGFBP1, PDGF, cyclin D1, MYC, and BIRC5. ${ }^{45}$ In the present study, two KLF5 and YAP common target genes were identified (FGFBP1 and ITGB2). In liver-specific YAP transgenic mice, the FGFBP1 mRNA level was increased 151-fold. ${ }^{4}$ Although FGF-BP has been implicated in promoting cell proliferation, survival, and tumor growth, ${ }^{28,46}$ we speculate that $Y A P$ regulates the transcription of a set of target genes (in addition to FGFBP1 and ITGB2) through KLF5. According to published microarray results, 4,27,38 PDGF, BIRC5, MYC, TIMP2, DUSP1/MKP1, TGFA, and EXT1 are potentially regulated by both KLF5 and YAP. Genome-wide 

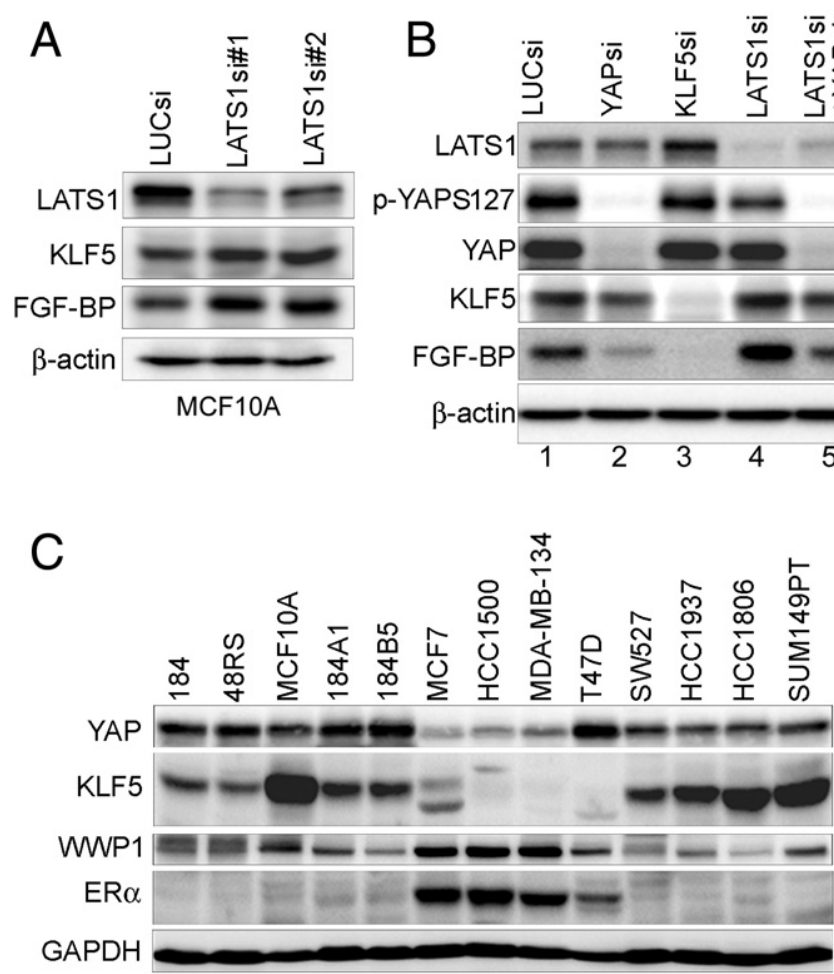
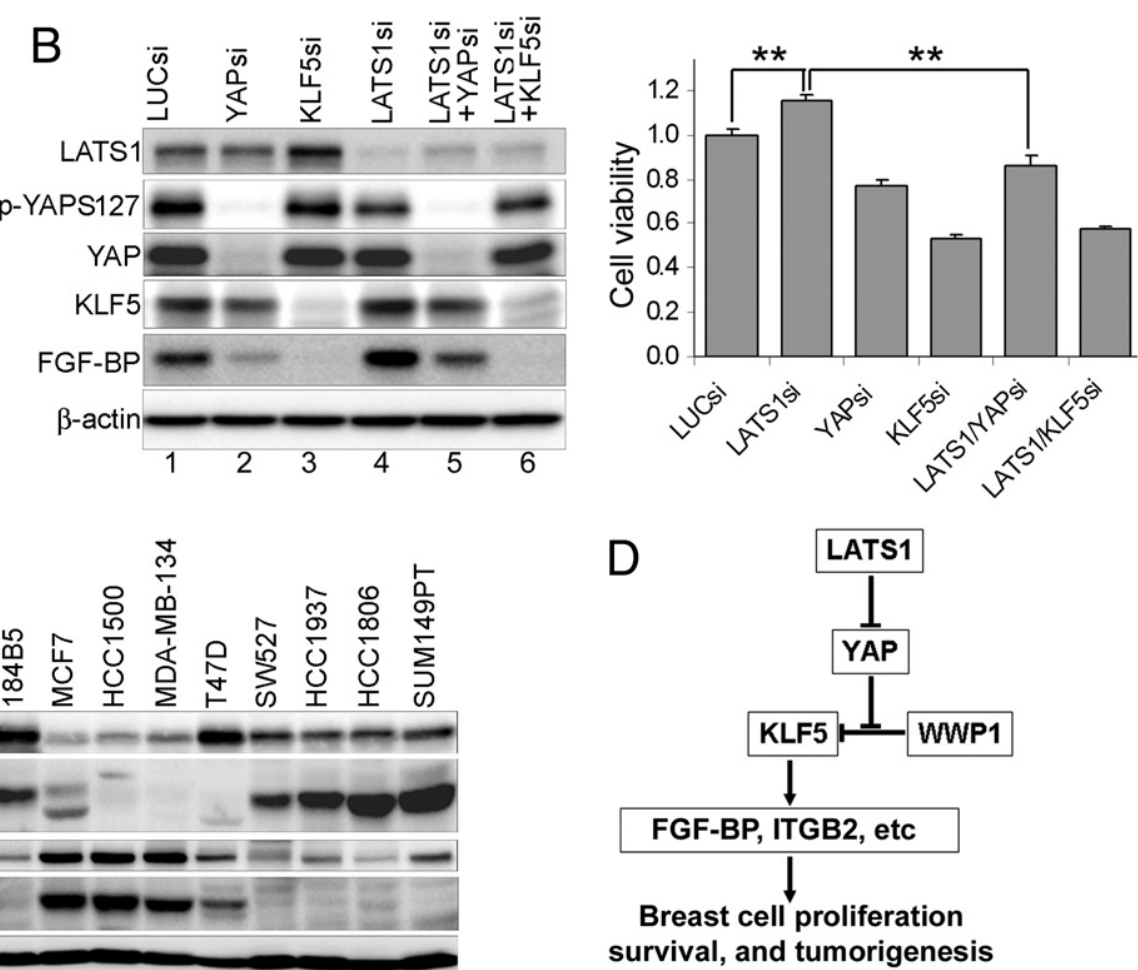

Figure 6. KLF5 is suppressed by LATS1 through YAP in MCF10A cells. A: LATS1 knockdown by two different siRNAs increased KLF5 and FGF-BP protein levels. For quantification of the relative KLF5 protein levels, see Supplemental Figure S5A (available at http://ajp.amjpathol.org). B: LATS1 suppressed the expression of KLF5 and FGF-BP through YAP. LATS1 knockdown decreased pYAP-S127 protein levels (lane 4 versus lane 1), and YAP depletion rescued LATS1 siRNA-induced increases in KLF5 and FGF-BP (lane 5 versus lane 4) (left panel). For quantitative data, see Supplemental Figure S5B (available at bttp://ajp.amipathol.org). In addition, LATS1 knockdown increased cell growth compared with LUC $_{\mathrm{si}}(P=0.002)$, as determined by the SRB assay, and YAP depletion blocked LATS1 siRNA-induced growth increase (right panel) $(P=0.0006) .{ }^{* * *} P<0.01$. C: Both YAP and KLF5 were expressed predominately in ER $\alpha^{-}$breast cell lines, excepting T47D, in which YAP but not KLF5 was expressed at a high level. WWP1 was expressed predominately in ER $\alpha^{+}$breast cell lines. D: A model illustrating the mechanism by which YAP functions partially through KLF5 in breast cells. The E3 ubiquitin ligase WWP1 targets KLF5 for degradation by binding to the KLF5 PY motif. YAP binds to KLF5 and protects KLF5 from WWP1-mediated degradation. The YAP upstream protein kinase LATS1 suppresses KLF5 through YAP. YAP and KLF5 increase the transcription of FGFBP1, ITGB2, and other downstream target genes, promoting breast cell proliferation, survival, and tumorigenesis.

studies will be required to identify all YAP target genes regulated through KLF5 in breast cells and in other scenarios.

KLF5 and YAP appear to have similar expression patterns and oncogenic functions in breast cancer. Both KLF5 and YAP tended to be expressed in ER $\alpha^{-}$but not $\mathrm{ER} \alpha^{+}$breast cell lines (Figure 6), although results need to be further validated in breast tumors by IHC. Knockdown of either KLF5 or YAP suppressed breast cell proliferation, survival, and tumor growth (Figures 4 and 5). For the first time we demonstrate that inhibition of KLF5 or YAP suppressed breast tumor growth in vivo. These results are consistent with previous studies in which YAP is suggested to be an oncoprotein in breast cells. ${ }^{5,12,15}$ Additionally, both KLF5 and YAP promoted colon cancer ${ }^{47,48}$ and maintained embryonic stem cell self-renewal. ${ }^{33,34}$ The oncogenic role of YAP has been suggested in a variety of cancers, including liver cancer, ${ }^{3}$ ovarian cancer, ${ }^{49}$ and lung cancer. ${ }^{50}$ Whether KLF5 has a similar role in these cancer types requires further investigation.

YAP has also been suggested to be a tumor suppressor in breast cancer ${ }^{40}$ and in head and neck squamous cell carcinomas. ${ }^{51}$ YAP has also been shown to promote apoptosis through potentiating the proapoptotic activities of p73, ZO-2, and EGR-1 in response to DNA damage. ${ }^{18,19,52,53}$ Whether YAP has a context-dependent role in breast cancer and other cancers needs to be validated by in vivo studies.

Liver-specific YAP transgenic mice exhibited increased organ size and induced hepatocellular carcinoma. ${ }^{4,14}$ Additionally, heterozygous deletion of Yap rescued Nf2 knockout-induced hepatocellular carcinoma. ${ }^{54}$ Yap null mice were deficient for dextran sodium sulfate-induced intestinal regeneration. ${ }^{48}$ Similarly, heterozygous KIf5 knockout mice exhibited reduced bacteria Citrobacter rodentium-induced crypt cell proliferation ${ }^{47}$ and $\mathrm{Apc}(\mathrm{Min})$ mutation-induced intestinal adenoma. ${ }^{42}$ The findings from these in vivo genetic studies suggest that YAP and KLF5 are potential oncoproteins in tumorigenesis. Whether YAP functions through KLF5 in vivo requires further investigation using transgenic mouse models.

In conclusion, YAP stabilized the KLF5 protein by competing with WWP1, attenuated WWP1-mediated KLF5 ubiquitination and degradation, and increased the KLF5 activities of inducing gene expression and promoting breast cell growth and survival. KLF5 could be an important YAP partner transcription factor mediating the Hippo tumor suppressor pathway. The LATS1-YAPKLF5 signaling axis may provide new targets for breast cancer treatment. 


\section{Acknowledgments}

We thank Dr. Kun-Liang Guan (University of California at San Diego) for the YAP constructs, Dr. Jin-Tang Dong (Emory University) for WWP1 and KLF5 antibodies, and Dr. Jiliang Zhou (Albany Medical College) for stimulating discussion.

\section{References}

1. Wang K, Degerny $\mathrm{C}, \mathrm{Xu}$ M, Yang XJ: YAP, TAZ, and Yorkie: a conserved family of signal-responsive transcriptional coregulators in animal development and human disease. Biochem Cell Biol 2009, 87:77-91

2. Zhao B, Li L, Lei Q, Guan KL: The Hippo-YAP pathway in organ size control and tumorigenesis: an updated version. Genes Dev 2010, 24:862-874

3. Zender L, Spector MS, Xue W, Flemming P, Cordon-Cardo C, Silke J, Fan ST, Luk JM, Wigler M, Hannon GJ, Mu D, Lucito R, Powers S, Lowe SW: Identification and validation of oncogenes in liver cancer using an integrative oncogenomic approach. Cell 2006, 125:12531267

4. Dong J, Feldmann G, Huang J, Wu S, Zhang N, Comerford SA, Gayyed MF, Anders RA, Maitra A, Pan D: Elucidation of a universal size-control mechanism in Drosophila and mammals. Cell 2007, 130: $1120-1133$

5. Overholtzer M, Zhang J, Smolen GA, Muir B, Li W, Sgroi DC, Deng CX, Brugge JS, Haber DA: Transforming properties of YAP, a candidate oncogene on the chromosome 11q22 amplicon. Proc Natl Acad Sci USA 2006, 103:12405-12410

6. Zhang X, George J, Deb S, Degoutin JL, Takano EA, Fox SB; AOCS Study group, Bowtell DD, Harvey KF: The Hippo pathway transcriptional co-activator. YAP, is an ovarian cancer oncogene, Oncogene 2011, 30:2810-2822

7. Xu MZ, Yao TJ, Lee NP, Ng IO, Chan YT, Zender L, Lowe SW, Poon $\mathrm{RT}$, Luk JM: Yes-associated protein is an independent prognostic marker in hepatocellular carcinoma. Cancer 2009, 115:4576-4585

8. Xu MZ, Chan SW, Liu AM, Wong KF, Fan ST, Chen J, Poon RT, Zender L, Lowe SW, Hong W, Luk JM: AXL receptor kinase is a mediator of YAP-dependent oncogenic functions in hepatocellular carcinoma. Oncogene 2011, 30:1229-1240

9. Muramatsu T, Imoto I, Matsui T, Kozaki K, Haruki S, Sudol M, Shimada Y, Tsuda H, Kawano T, Inazawa J: YAP is a candidate oncogene for esophageal squamous cell carcinoma. Carcinogenesis 2011, 32 : 389-398

10. Hsu JH, Lawlor ER: BMl-1 suppresses contact inhibition and stabilizes YAP in Ewing sarcoma. Oncogene 2011, 30:2077-2085

11. Zhao B, Lei QY, Guan KL: The Hippo-YAP pathway: new connections between regulation of organ size and cancer. Curr Opin Cell Biol 2008, 20:638-646

12. Zhang J, Smolen GA, Haber DA: Negative regulation of YAP by LATS1 underscores evolutionary conservation of the Drosophila Hippo pathway. Cancer Res 2008, 68:2789-2794

13. Zhao B, Kim J, Ye X, Lai ZC, Guan KL: Both TEAD-binding and WW domains are required for the growth stimulation and oncogenic transformation activity of yes-associated protein. Cancer Res 2009, 69: 1089-1098

14. Camargo FD, Gokhale S, Johnnidis JB, Fu D, Bell GW, Jaenisch R, Brummelkamp TR: YAP1 increases organ size and expands undifferentiated progenitor cells [Erratum appeared in Curr Biol 2007, 17: 2094]. Curr Biol 2007, 17:2054-2060

15. Zhao B, Ye X, Yu J, Li L, Li W, Li S, Yu J, Lin JD, Wang CY, Chinnaiyan AM, Lai ZC, Guan KL: TEAD mediates YAP-dependent gene induction and growth control. Genes Dev 2008, 22:1962-1971

16. Zhang H, Liu CY, Zha ZY, Zhao B, Yao J, Zhao S, Xiong Y, Lei QY, Guan KL: TEAD transcription factors mediate the function of TAZ in cell growth and epithelial-mesenchymal transition. J Biol Chem 2009, 284:13355-13362

17. Basu S, Totty NF, Irwin MS, Sudol M, Downward J: Akt phosphorylates the Yes-associated protein, YAP, to induce interaction with 14-
3-3 and attenuation of p73-mediated apoptosis. Mol Cell 2003, $11: 11-23$

18. Levy D, Adamovich Y, Reuven N, Shaul Y: The Yes-associated protein 1 stabilizes p73 by preventing Itch-mediated ubiquitination of p73. Cell Death Differ 2007, 14:743-751

19. Zagurovskaya M, Shareef MM, Das A, Reeves A, Gupta S, Sudol M, Bedford MT, Prichard J, Mohiuddin M, Ahmed MM: EGR-1 forms a complex with YAP-1 and upregulates Bax expression in irradiated prostate carcinoma cells. Oncogene 2009, 28:1121-1131

20. Omerovic J, Puggioni EM, Napoletano S, Visco V, Fraioli R, Frati L, Gulino A, Alimandi M: Ligand-regulated association of ErbB-4 to the transcriptional co-activator YAP65 controls transcription at the nuclear level. Exp Cell Res 2004, 294:469-479

21. Aqeilan RI, Donati V, Palamarchuk A, Trapasso F, Kaou M, Pekarsky $Y$, Sudol M, Croce CM: WW domain-containing proteins, WWOX and YAP, compete for interaction with ErbB-4 and modulate its transcriptional function. Cancer Res 2005, 65:6764-6772

22. Muraoka-Cook RS, Sandahl M, Hunter D, Miraglia L, Earp HS 3rd: Prolactin and ErbB4/HER4 signaling interact via Janus kinase 2 to induce mammary epithelial cell gene expression differentiation. Mol Endocrinol 2008, 22:2307-2321

23. Vitolo MI, Anglin IE, Mahoney WM Jr, Renoud KJ, Gartenhaus RB, Bachman KE, Passaniti A: The RUNX2 transcription factor cooperates with the YES-associated protein, YAP65, to promote cell transformation. Cancer Biol Ther 2007, 6:856-863

24. Zhang X, Milton CC, Humbert PO, Harvey KF: Transcriptional output of the Salvador/Warts/Hippo pathway is controlled in distinct fashions in Drosophila melanogaster and mammalian cell lines. Cancer Res 2009, 69:6033-6041

25. Ben-Porath I, Thomson MW, Carey VJ, Ge R, Bell GW, Regev A, Weinberg RA: An embryonic stem cell-like gene expression signature in poorly differentiated aggressive human tumors. Nat Genet 2008, 40:499-507

26. Tong D, Czerwenka K, Heinze G, Ryffel M, Schuster E, Witt A, Leodolter S, Zeillinger R: Expression of KLF5 is a prognostic factor for disease-free survival and overall survival in patients with breast cancer. Clin Cancer Res 2006, 12:2442-2448

27. Chen C, Benjamin MS, Sun X, Otto KB, Guo P, Dong XY, Bao Y, Zhou $Z$, Cheng $X$, Simons JW, Dong JT: KLF5 promotes cell proliferation and tumorigenesis through gene regulation in the TSU-Pr1 human bladder cancer cell line. Int J Cancer 2006, 118:1346-1355

28. Zheng HQ, Zhou Z, Huang J, Chaudhury L, Dong JT, Chen C: Krüppel-like factor 5 promotes breast cell proliferation partially through upregulating the transcription of fibroblast growth factor binding protein 1. Oncogene 2009, 28:3702-3713

29. Liu R, Zheng HQ, Zhou Z, Dong JT, Chen C: KLF5 promotes breast cell survival partially through fibroblast growth factor-binding protein 1-pERK-mediated dual specificity MKP-1 protein phosphorylation and stabilization. J Biol Chem 2009, 284:16791-16798

30. Chen C, Sun X, Ran Q, Wilkinson KD, Murphy TJ, Simons JW, Dong $\mathrm{JT}$ : Ubiquitin-proteasome degradation of KLF5 transcription factor in cancer and untransformed epithelial cells. Oncogene 2005, 24:33193327

31. Chen C, Sun X, Guo P, Dong XY, Sethi P, Cheng X, Zhou J, Ling J, Simons JW, Lingrel JB, Dong JT: Human Krüppel-like factor 5 is a target of the E3 ubiquitin ligase WWP1 for proteolysis in epithelial cells. J Biol Chem 2005, 280:41553-41561

32. Zhao D, Zheng HQ, Zhou Z, Chen C: The Fbw7 tumor suppressor targets KLF5 for ubiquitin-mediated degradation and suppresses breast cell proliferation. Cancer Res 2010, 70:4728-4738

33. Parisi S, Passaro F, Aloia L, Manabe I, Nagai R, Pastore L, Russo T: $\mathrm{Klf5}$ is involved in self-renewal of mouse embryonic stem cells. J Cell Sci 2008, 121:2629-2634

34. Lian I, Kim J, Okazawa H, Zhao J, Zhao B, Yu J, Chinnaiyan A, Israel MA, Goldstein LS, Abujarour R, Ding S, Guan KL: The role of YAP transcription coactivator in regulating stem cell self-renewal and differentiation. Genes Dev 2010, 24:1106-1118

35. Chen C, Zhou Z, Ross JS, Zhou W, Dong JT: The amplified WWP1 gene is a potential molecular target in breast cancer. Int $\mathrm{J}$ Cancer 2007, 121:80-87

36. Chen C, Zhou Y, Zhou Z, Sun X, Otto KB, Uht RM, Dong JT: Regulation of KLF5 involves the Sp1 transcription factor in human epithelial cells. Gene 2004, 330:133-142 
37. Chang BL, Cramer SD, Wiklund F, Isaacs SD, Stevens VL, Sun J, Smith S, Pruett K, Romero LM, Wiley KE, Kim ST, Zhu Y, Zhang Z, Hsu FC, Turner AR, Adolfsson J, Liu W, Kim JW, Duggan D, Carpten J, Zheng SL, Rodriguez C, Isaacs WB, Grönberg H, Xu J: Fine mapping association study and functional analysis implicate a SNP in MSMB at $10 \mathrm{q} 11$ as a causal variant for prostate cancer risk. Hum Mol Genet 2009, 18:1368-1375

38. Hao Y, Chun A, Cheung K, Rashidi B, Yang X: Tumor suppressor LATS1 is a negative regulator of oncogene YAP. J Biol Chem 2008 , 283:5496-5509

39. Chen C, Zhou Z, Sheehan CE, Slodkowska E, Sheehan CB, Boguniewicz A, Ross JS: Overexpression of WWP1 is associated with the estrogen receptor and insulin-like growth factor receptor 1 in breast carcinoma. Int J Cancer 2009, 124:2829-2836

40. Yuan M, Tomlinson V, Lara R, Holliday D, Chelala C, Harada T, Gangeswaran R, Manson-Bishop C, Smith P, Danovi SA, Pardo O, Crook T, Mein CA, Lemoine NR, Jones LJ, Basu S: Yes-associated protein (YAP) functions as a tumor suppressor in breast. Cell Death Differ 2008, 15:1752-1759

41. Nandan MO, McConnell BB, Ghaleb AM, Bialkowska AB, Sheng $H$, Shao J, Babbin BA, Robine S, Yang VW: Krüppel-like factor 5 mediates cellular transformation during oncogenic KRAS-induced intestinal tumorigenesis. Gastroenterology 2008, 134:120-130

42. McConnell BB, Bialkowska AB, Nandan MO, Ghaleb AM, Gordon FJ, Yang VW: Haploinsufficiency of Krüppel-like factor 5 rescues the tumor-initiating effect of the Apc(Min) mutation in the intestine. Cancer Res 2009, 69:4125-4133

43. Guo P, Dong XY, Zhao K, Sun X, Li Q, Dong JT: Opposing effects of KLF5 on the transcription of MYC in epithelial proliferation in the context of transforming growth factor beta. J Biol Chem 2009, 284: 28243-28252

44. Zhang J, Ji JY, Yu M, Overholtzer M, Smolen GA, Wang R, Brugge JS, Dyson NJ, Haber DA: YAP-dependent induction of amphiregulin identifies a non-cell-autonomous component of the Hippo pathway. Nat Cell Biol 2009, 11:1444-1450
45. Dong JT, Chen C: Essential role of KLF5 transcription factor in cell proliferation and differentiation and its implications for human diseases. Cell Mol Life Sci 2009, 66:2691-2706

46. Tassi E, Henke RT, Bowden ET, Swift MR, Kodack DP, Kuo AH, Maitra A, Wellstein A: Expression of a fibroblast growth factor-binding protein during the development of adenocarcinoma of the pancreas and colon. Cancer Res 2006, 66:1191-1198

47. McConnell BB, Klapproth JM, Sasaki M, Nandan MO, Yang VW: Krüppel-like factor 5 mediates transmissible murine colonic hyperplasia caused by Citrobacter rodentium infection. Gastroenterology 2008, 134:1007-1016

48. Cai J, Zhang N, Zheng Y, de Wilde RF, Maitra A, Pan D: The Hippo signaling pathway restricts the oncogenic potential of an intestinal regeneration program. Genes Dev 2010, 24:2383-2388

49. Hall CA, Wang R, Miao J, Oliva E, Shen X, Wheeler T, Hilsenbeck SG, Orsulic S, Goode S: Hippo pathway effector Yap is an ovarian cancer oncogene. Cancer Res 2010, 70:8517-8525

50. Wang Y, Dong Q, Zhang Q, Li Z, Wang E, Qiu X: Overexpression of yes-associated protein contributes to progression and poor prognosis of non-small-cell lung cancer. Cancer Sci 2010, 101:1279-1285

51. Ehsanian R, Brown M, Lu H, Yang XP. Pattatheyil A, Yan B, Duggal P, Chuang R, Doondeea J, Feller S, Sudol M, Chen Z, Van Waes C: YAP dysregulation by phosphorylation or DeltaNp63-mediated gene repression promotes proliferation, survival and migration in head and neck cancer subsets. Oncogene 2010, 29:6160-6171

52. Lapi E, Di Agostino S, Donzelli S, Gal H, Domany E, Rechavi G, Pandolfi PP, Givol D, Strano S, Lu X, Blandino G: PML, YAP, and p73 are components of a proapoptotic autoregulatory feedback loop. Mol Cell 2008, 32:803-814

53. Oka T, Sudol M: Nuclear localization and pro-apoptotic signaling of YAP2 require intact PDZ-binding motif. Genes Cells 2009, 14:607615

54. Zhang N, Bai H, David KK, Dong J, Zheng Y, Cai J, Giovannini M, Liu $P$, Anders RA, Pan D: The Merlin/NF2 tumor suppressor functions through the YAP oncoprotein to regulate tissue homeostasis in mammals. Dev Cell 2010, 19:27-38 